\title{
HISTORIA, CONCEPTOS Y PROPUESTAS EN LA TERAPIA OCUPACIONAL SOCIAL DE BRASIL
}

HISTORY, CONCEPTS AND PROPOSALS ON SOCIAL OCCUPATIONAL THERAPY IN

BRAZIL

\section{Roseli Esquerdo Lopes ${ }^{1}$, Ana Paula S. Malfitano ${ }^{2}$, Carla Regina Silva ${ }^{3}$, Patrícia L. O. Borba $^{4}$}

\begin{abstract}
Resumen
Este artículo presenta la constitución histórica de la Terapia Ocupacional Social en Brasil, fruto de un proceso que la configuró como un campo complejo y de fronteras, el cual desarrolla tecnologías y acciones que buscan lidiar con cuestiones provenientes de las desigualdades que emergen de las contradicciones sociales, dentro de las sociedades capitalistas. Se discute, inicialmente, lo que fueron las variaciones de comprensión teórico-metodológica en el discurso profesional en torno a "lo social", para después abordar las perspectivas teóricas básicas que fundamentaron y sustentaron las propuestas de esa área. De este modo, se relatan algunas vertientes -nacionales e internacionales- de autores que han valorado las demandas sociales para la terapia ocupacional, así como de autores que defienden una terapia ocupacional social, radicados en Brasil. Y por último, utilizando parte de nuestras experiencias en el Proyecto METUIA, el cual reúne actividades de enseñanza, investigación y extensión en esa área, y que se desarrolla en cuatro diferentes universidades brasileñas, se presenta cómo hemos afrontado las cuestiones relacionadas con un determinado grupo: la juventud pobre urbana; y trabajado en la producción de tecnologías sociales en terapia ocupacional social que, desde nuestro punto de vista, han sido capaces de fomentar nuevas posibilidades de actuación terapéutico-ocupacional, integrando y articulando acciones de alcance macro e microsocial.
\end{abstract}

\section{Palabras clave:}

Terapia Ocupacional; Propuestas y Tendencias en Terapia Ocupacional; Terapia Ocupacional Social; Historia; Cuestiones Sociales.

1 Maestría y Doctorado en Educación, Profesora Titular del Departamento de Terapia Ocupacional de la Universidad Federal de São Carlos (UFSCar). Dirección: METUIA Laboratorio, Departamento de Terapia Ocupacional UFSCar. Rod. Washington Luís, km 235 - SP-310. Caixa Postal 676. São Carlos, SP, Brasil. 13.565-905. +551633518640. relopes@ufscar.br

2 Maestría en Educación y Doctorado en Salud Pública, Profesora del Departamento de Terapia Ocupacional de la Universidad Federal de São Carlos (UFSCar). Dirección: METUIA Laboratorio, Departamento de Terapia Ocupacional UFSCar. Rod. Washington Luís, km 235 - SP310. Caixa Postal 676. São Carlos, SP, Brasil. 13.565-905. +551633066623. amalfitano@ufscar.br

3 Maestría y Doctorado en Educación, Profesora del Departamento de Terapia Ocupacional de la Universidad Federal de São Carlos (UFSCar). Dirección: METUIA Laboratorio, Departamento de Terapia Ocupacional UFSCar. Rod. Washington Luís, km 235 - SP-310. Caixa Postal 676. São Carlos, SP, Brasil. 13.565-905. +551633518743. carlars@ufscar.br

4 Maestría y Doctorado en Educación, Profesora del Departamento de Salud, Educación y Sociedad de la Universidad Federal de São Paulo, Santos. Dirección: Departamento de Salud, Educación y Sociedad de la Unifesp. Rua Silvia Jardim, 136 Santos, SP, Brasil. CEP 11015-020. +551338783700. patricialemeborba@hotmail.com 


\begin{abstract}
This article presents a historical constitution of Social Occupational Therapy in Brazil, resulting from a process that has configured it as a complex field with limitations, which develops technologies and actions that aim to cope with issues arising from the inequalities that emerge from social contradictions in capitalist societies. Initially, we discuss the variations of the theoretical and methodological understanding in professional discourse regarding the social aspect; after that, we outline the basic theoretical perspectives that have supported and sustained the propositions of this area. We report some aspects - national and international - presented by authors that have underlined social demands for occupational therapy, as well as those presented by authors living in Brazil who advocate a social occupational therapy. Finally, taking part of our experiences with the METUIA Project, which gathers activities of education, research and extension in this field, and it is developed in four different Brazilian universities, we present how we have coped with issues related to a particular group - the poor urban youth, and worked in the production of social technologies on social occupational therapy which, from our standpoint, have been able to foster new opportunities for therapeutic-occupational activities, integrating and articulating actions of macro and micro scopes.
\end{abstract}

\title{
Keywords:
}

Occupational Therapy/Trends, Social Occupational Therapy/History, Social Occupational Therapy/Propositions, Occupational Therapy/Social Issues.

\section{TERAPIA OCUPACIONAL Y LAS VARIACIONES}

\section{DEL DISCURSO EN TORNO DE LO SOCIAL}

La formación de terapeutas ocupacionales en Brasil data de mitad de la década de 1950 en Rio de Janeiro y en São Paulo, siendo esta última la ciudad adonde se formalizó, frente al Ministerio de Educación, en el Instituto de Rehabilitación de la Facultad de Medicina de la Universidad de São Paulo. Eran cursos dirigidos a la enseñanza de los Ilamados "profesionales de la rehabilitación", sobretodo, fisioterapeutas y terapeutas ocupacionales, a partir de los convenios con la ONU y la UNICEF. Esa formación estaba basada en la rehabilitación en salud, en sentido amplio, aunque bastante enfocada en la rehabilitación para los accidentados laborales, articulada a una perspectiva de la disfunción y de la deficiencia física; ciertamente, también se colocaba, dentro del histórico de la configuración de la profesión en los países del norte, la dimensión de la psiquiatría, en el caso de la terapia ocupacional, y la rehabilitación para la vida en "ocupación" (Lopes, 1990; Soares, 1991).

Este eje orientado para la rehabilitación va a ser importante y controvertido para los terapeutas ocupa- cionales brasileños, especialmente en su identidad y desempeño profesional, tanto focalizado en la función en sí misma, como en el desempeño funcional y/o ocupacional. Sólo a partir de mediados de la década de 1970 se pueden identificar intervenciones de terapeutas ocupacionales en el campo social. Las inserciones de estos profesionales ocurrieron, en primer lugar, en cárceles y en instituciones para niños y adolescentes pobres y abandonados, que cometieron un "acto infractor", un término todavía muy debatido en la época (Pinto, 1990). Es importante señalar que las primeras acciones entendidas dentro del campo social en Brasil se dieron durante la dictadura militar (1964-1985), en las instituciones totales y bajo el referencial de políticas públicas represivas (Barros, Lopes y Galheigo, 2007).

De esta manera, estas primeras incursiones en la década de 1970, orientadas a actitudes/comportamientos de individuos y grupos considerados como desviados, desajustados, marginales, tanto en el plano psicosocial como en el plano macrosocial, caracterizaron las acciones de aquel período, igualmente marcadas por las impugnaciones a ese tipo de entendimiento, pero que, todavía, eran absolutamente reprimidas. 
Figura 1:

Homenaje de Hélio Oiticica a Cara de Cavalo5, Brasil, 1965-1968

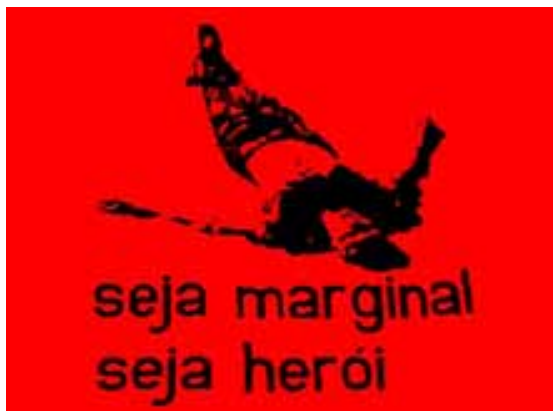

Ese "sea marginal, sea héroe", conforme Figura 1, que tomó diversas formas entre 1965-1968, representa parte de los cuestionamientos posibles en el Brasil de ese momento, en relación a la sociedad represiva y respetuosa con el orden estructural capitalista. Ese es el contexto social y político donde se fueron formando los equipos multiprofesionales y en donde se constituyeron sus acciones en el campo social, siendo el terapeuta ocupacional uno de los técnicos que se incorporaron más tardíamente (Barros, Lopes y Galheigo, 2007).

Se debe considerar el hecho de que la terapia ocupacional brasileña tiene grandes dificultades con la reconstrucción de su historia antes de la década de 1980, momento anterior al que designamos como profesionalización de la terapia ocupacional, la cual ocurre en Brasil, efectivamente, a finales de la década de 1970, cuando pasamos a dar importancia y a registrar documentos junto con la propia historia de la terapia ocupacional; una historia que todavía tiene que estudiarse, de modo más sistematizado y con metodologías propias.

Por lo tanto, aunque no afirmaremos que no hay ningún indicio anterior de acciones del terapeuta ocupacional en el campo social, sí que observamos que

\footnotetext{
Manoel Moreira (1941-1964) ligado a la contravención en Rio de Janeiro (juego de números, prostitución), acusado del asesinato de Milton Le Cocq, detective ligado a la policía carioca, fue la primera víctima, en octubre de 1964, de la Scuderie Le Cocq, organización paramilitar clandestina creada para vengar la muerte del detective. Un año después de la muerte, Hélio Oiticica creó la obra "Homenaje a Cara de Cavalo", una caja envuelta por telas, cuyas paredes internas se cubrieron con fotografías del criminal asesinado. En 1968, él rendiría otro tributo al amigo, con el poema-bandera "Sea marginal, sea héroe". La Scuderie inspiró el surgimiento del Escuadrón de la Muerte en Brasil (Ventura, 1994).
}

la primera publicación sobre la temática aparece en los Anales del "V Encuentro Científico Paulista de Terapeutas Ocupacionales", en 1979, con autoría de la terapeuta ocupacional Jussara de Mesquita Pinto, y que traía el relato de su actuación en la ciudad de São Paulo en una institución para adolescentes que cometieron algún acto infractor, utilizando el término "terapia ocupacional social", en el abordaje profesional junto a chicas en situación de privación de libertad (Pinto, 1979).

Además de ese texto, el grupo de profesionales, formados en 1979, del Curso de Pregrado en Terapia Ocupacional de la Universidad de São Paulo (USP) puede abrir e insertarse en prácticas profesionalizantes de terapia ocupacional social, fruto de una organización colectiva entre profesionales supervisores y estudiantes que, por ese motivo, lo solicitaron.

En ese contexto, el surgimiento de la terapia ocupacional social en Brasil apareció con ese grupo de terapeutas ocupacionales que estaba en los nuevos campos de prácticas y que tenía como base algunas experiencias en curso, aliándose a las vivencias de la aplicación de formulaciones propias de la terapia ocupacional, especialmente el uso de actividades y dinámicas grupales, y aliándose a aquella nueva situación y a sus demandas. Era importante, para el área, ser aceptada y pasar a integrar el equipo técnico. De esa forma, se puede señalar que la acción profesional comenzó a suceder dentro de esos nuevos espacios sin una mayor reflexión, ya que, en ese primer momento, tenían la intención de ocupar espacio, de estar allí de algún modo y ser reconocida. (Barros, Lopes y Galheigo, 2007). En relación a ese aparato de control, la crítica era menor y se orientaba hacia proposiciones que derivaban de las denominadas "teorías de la marginalidad" (Escorel, 1999), en la que el técnico ocupaba un papel central, casi siempre desde una perspectiva de "adaptador social" (Galheigo, 1997), lo que, de hecho, no es muy diferente de algunas acciones que se mantienen en la actualidad, con la diferencia de que, en aquel período, la estructura represiva de los servicios no necesitaba disimularse.

De entre las instituciones y políticas ligadas al cercenamiento de la libertad, hay muchos ejemplos orientados para la adolescencia y juventud, dado que el "asilamiento" de niños y jóvenes era socialmente bastante aceptado, y su práctica reunía "abrigo" y control, desde el inicio del siglo en Brasil, aliados con la educación para y por el trabajo (Rafante y Lopes, 2009).

También existía el trabajo de terapeutas ocupacionales junto a la infancia pobre asilada, principalmente en sus años iniciales, en instituciones públicas o en 
instituciones privadas, especialmente confesionales y filantrópicas. En este caso, del niño pequeño pobre, la intervención terapéutica-ocupacional tenía, por un lado, una perspectiva social focalizada en la oferta de bienes imprescindibles para la existencia material y del desarrollo de ese niño. Sin embargo, partiendo desde una perspectiva psicopedagógica, lo que predominantemente señalaba la práctica de los profesionales era la búsqueda de un desarrollo "normal".

Un tema candente, relacionado con la pobreza en Brasil, era cuidar efectivamente de los niños pequeños; sin embargo, tanto en aquella época como hoy en día, el cuidado de los jóvenes adolescentes estaba vinculado, antes de nada, al control y a la represión, principalmente de los jóvenes mestizos, negros y pobres.

Así, a mediados de la década de 1970, la terapia ocupacional social comenzó a adquirir contornos propios bajo la égida de un régimen autoritario, que imponía un sistema disciplinario y segregacionista, aunque mistificado por el tecnicismo que, al conceder el poder a los técnicos, buscaba garantizar la conformidad con la violencia de una sociedad profundamente desigual.

La crítica a ese modelo, que comienza a destacarse a inicios de la década de 1990 por parte de organizaciones y movimientos de la sociedad civil, en el escenario político, es acompañada del debate académico que recolocaba la cuestión de la marginalidad social, y de las teorías que prevén esa perspectiva, sobre la óptica del conflicto social en una sociedad basada en la propiedad privada y en la explotación de unos sobre otros (Barros, Lopes y Galheigo, 2007).

En ese mismo período, entre finales de la década de 1970 e inicios de 1980, una pauta importante para la terapia ocupacional brasileña fue la de los debates de las estructuras formales de la profesionalización (organismos reguladores y fiscalizadores) y la de la formación (currículo mínimo a ser exigido para los cursos de pregrado), los cuales no podrían dejar dudas en cuanto al carácter de su nivel académico superior, ya que en la reglamentación de la profesión y en el currículo de 1969 aparecían lagunas que dejaban márgenes para una comprensión de una profesionalización que demandaba una enseñanza de nivel técnico, intermediario y no superior (Lopes, 1990).

En ese debate, que era amplio, se envolvían varios sectores y perspectivas. Desde la perspectiva de la terapia ocupacional, estaban los sectores que actuaban en el ámbito social y/o que militaban por una terapia ocupacional crítica, ampliándose también espacios en la defensa de una formación competente, tanto a nivel técnico como político.

En la terapia ocupacional social, denominación de un campo de estudio y de intervención, las demandas implicadas en esa temática, la necesidad de conocimiento y postura crítica con relación a determinados posicionamientos ideológicos, la concepción del hombre y de la sociedad, la definición de modelos de intervención, etc. todo eso componía un debate que siempre generaba conflictos. Al hilo de las reformas curriculares, se discute si la designación de las disciplinas y/o programas de enseñanza sería "Terapia Ocupacional Social" o "Terapia Ocupacional en el Campo Social", se discute si serían esas disciplinas las que acogerían, en pregrado, ese cuerpo de conocimiento. ¿Qué es lo que se supondría cuando se supondría hablar de una terapia ocupacional aplicada a las disfunciones sociales? ¿Qué son las disfunciones sociales? ¿La sociedad es la que tiene las disfunciones? ¿Los individuos en la sociedad son los que tienen las disfunciones? Ese era el debate. ¿La terapia ocupacional aplicada a las condiciones sociales se dirigiría hacia la falta de condiciones sociales? ¿Se dirigiría hacia las condiciones dadas?

En fin, ese fue un proceso rico, importante y contradictorio entre nosotros; a partir de él y de lo que aprendieron los profesionales del área que vivenciaron ese momento, se configuró su cuerpo teórico y metodológico; dicho cuerpo se constituye conjuntamente con la crítica y reformulación de la denominación de asignaturas, la búsqueda de aquello que pudiese convertirse en el referencial teórico más apropiado para fundamentar la acción terapéutico-ocupacional en el campo social.

La noción de "rehabilitación social" era una temática relevante y pertinente. Desde la epidemiologia social, en dicha perspectiva se colocan una serie de problemas. Diversos profesionales "miran" la cuestión social presente en lo cotidiano de sus acciones, clamando por un posicionamiento para lidiar con ella; eso fue importante en el sector de la salud. La salud pública y la salud colectiva son sus ejemplos. Sin embargo, se percibe la necesidad de un diálogo con las ciencias humanas y sociales para entender los fenómenos sociales; se busca, durante la década de 1980, la fundamentación teórica y una crítica bien colocada, auxiliada por la historia y por la sociología, por sectores de la antipsiquiatría, de la lucha por la reforma sanitaria, por campos que generaban el debate y construían la crítica macrosocial a la asistencia. 
En este contexto, existía una crítica a los abordajes de la terapia ocupacional, una crítica a lo que efectivamente se hacía en la intervención, que estaba y está, aún mayoritariamente, focalizada en la "recuperación de la función".

La limitada adecuación de los modelos profesionales a esta realidad genera la siguiente polémica: ¿hay que elaborar un nuevo modelo específico - la terapia ocupacional social? [...] ¿O hay que implicarse en la propia concepción de la terapia ocupacional y buscar alternativas en ella misma? (Soares, 1991, p. 174).

Otro debate importante de la época ocurrió en torno de lo que serían los fundamentos de la terapia ocupacional. De entre otras iniciativas, un grupo de docentes de dos universidades del estado de São Paulo, a saber, de la Universidad Federal de São Carlos (UFSCar) y de la Pontificia Universidad de Campinas (PUC-Campinas), organizaron esa discusión sobre los métodos, campos e intervenciones (Pinto, 1987); entre sus resultados, por diferentes caminos, pero con aportes semejantes, Berenice Francisco y Jussara Pinto elaboraron lo que denominaron como "corrientes metodológicas en terapia ocupacional", buscando encuadrar, de acuerdo a una perspectiva de la producción del conocimiento, la terapia ocupacional y sus prácticas. Habría, entonces, una terapia ocupacional positivista, humanista o materialista-histórica (Francisco, 1988; Pinto, 1990). En ese momento, al hablar de transformación social se colocaba cualquier terapia ocupacional sobre la égida del materialismo-histórico; así, el hecho de preocuparse con los conflictos sociales no sería una cuestión de terapia ocupacional social, sino que sería un social totalizador que debería ser llevado a la comprensión de los terapeutas ocupacionales, desde el punto de vista del abordaje y de la visión de sujeto y de sociedad (Pinto, 1990).

Algunos terapeutas ocupacionales plantearon ese debate donde la realidad social se impondría a la práctica; sin embargo, de una forma hasta paradójica, esa discusión, ese énfasis "no social", tornó redundante una terapia ocupacional social, ya que, si ésta tiene que lidiar con las condiciones sociales, si todos los terapeutas ocupacionales se plantean la necesidad de cambio social, y si es importante considerar que había una identificación genuina con la propuesta de la transformación social, entonces, ¿cómo se hace una terapia ocupacional materialista-histórica? Esa era la pregunta y, en ese sentido, no tendría lugar una terapia ocupacional social, ni un campo de práctica y de estudio orientado a la cuestión social.

Tal perspectiva llevó a la exclusión de asignaturas de terapia ocupacional social en los currículos de pregrado o a la incorporación de su contenido en otras asignaturas de diferentes ámbitos. Por ejemplo, uno de ellos fue que lo "social" fuese trabajado tomándose el materialismo-histórico, la perspectiva transformadora de la sociedad, los fundamentos, las bases de la terapia ocupacional, de tal modo que toda y cualquier práctica derivaran de ellos. Aquellos terapeutas ocupacionales y docentes más identificados con esa visión abrazaron las asignaturas de ese espectro. Otro enfoque se elaboró entre los que buscaban colocar la óptica del desarrollo humano pleno, en el que sería posible trabajar por eso de modo socialmente referenciado.

También fue en ese período (década de 1980 e inicios de 1990) que la demanda de una terapia ocupacional profesionalizada, con un currículo e instituciones académicas que educasen adecuadamente a sus profesionales fue relativamente satisfecha en Brasil, en la que los docentes del área se capacitaban para la investigación y en la que a los terapeutas ocupacionales se les llamaba para integrar políticas públicas se constituyeran en la esfera social, con espacios concretos para desarrollar intervenciones que se situaban en campos específicos para producir conocimiento, acciones y asistencia, en la que la perspectiva de un campo social en terapia ocupacional tuvo un enorme resurgimiento.

En la década de 1990, se preguntaban, todavía, ¿qué lugar ocupa lo social en la terapia ocupacional? O ¿cuál es el papel del campo social en la terapia ocupacional? Los debates, las conversaciones y las diversas demandas del campo se van evidenciando; a pesar de las variaciones en torno del discurso de lo social en la terapia ocupacional, algunos profesionales permanecieron y/o pasaron a tomar para si la cuestión de la actuación y de la proposición del desarrollo de una terapia ocupacional social.

Al final de esa década, se recolocaría la cuestión social entre los terapeutas ocupacionales brasileños y la constitución del campo social en terapia ocupacional, derivado fuertemente de la problematización de la mediación necesaria entre salud y enfermedad en la acción profesional y de la proposición de una terapia ocupacional fuera del área de la salud (Barros, Ghirardi y Lopes, 2002, 2007). 
Tomándose la cuestión social, las perspectivas teóricas básicas sobre ella para reflexionar sobre las acciones profesionales, se pasa a la defensa y a la formulación de un trabajo en torno de una terapia ocupacional no sólo como una profesión "de la salud", sino como una profesión que puede mirar para los diferentes sectores/campos y pensar, proponer, desarrollar y discutir actuaciones profesionales en los mismos, permaneciendo la salud no sólo como un lugar importante, sino como un campo en coexistencia con otros, por ejemplo, el de la rehabilitación profesional, donde existe todo un diálogo presente, históricamente, en la terapia ocupacional con una discusión económico-social que a pesar de serle fundamental y necesaria, a veces, es poco considerada en los procesos de rehabilitación formateados por la salud (Ghirardi, 2012; Bregalda y Lopes, 2013).

De cierta forma, ese es un marco bastante importante en un momento que se plantea con mayor propiedad la capacitación de los docentes. Puede decirse que aquella discusión sobre los fundamentos de la profesión, todavía incipiente en la década de 1980, tuvo correspondencia y alcanzó su punto más alto con las trayectorias de profesionales que avanzaban y concluían sus maestrías; a finales de la década de 1990 se observó, relativamente, el inicio de un gran aumento de doctores entre los terapeutas ocupacionales brasileños.

Sin embargo, igualmente ese es el período en que Brasil y todo el mundo pasa por el liberalismo radical en la gestión de la esfera pública, con importantes repercusiones en la relación capital-trabajo y, por tanto, se agrava la cuestión social, cuyo enfrentamiento envolvió amplios sectores sociales y profesionales, entre los que están los terapeutas ocupacionales, en la busca de soluciones de lo que Castel (1998) y otros autores denominaron como la reconfiguración de la cuestión social a finales del siglo XX.

\section{Terapia Ocupacional Social: un Campo Complejo y de Fronteras}

La terapia ocupacional social en Brasil se desarrolló a partir de dos perspectivas teóricas complementarias; la primera tenía como parámetro el análisis de los procesos sociales, sobre todo desde finales de la década de 1970 hasta mediados 1980, una época de ebullición en torno de las demandas de la cuestión social; ésta no sólo era entendida como resultante de las relaciones entre capital-trabajo sino que también se refería a la construcción de la esfera pública movilizada por la sociedad civil, en un momento de "apertura política", aunque todavía impregnado por la restricción de esa propia esfera. A su vez, la transformación del Estado, producida por la lucha resultante de los movimientos sociales, se articulaba en una perspectiva democrática y de derecho en los límites del capitalismo.

Ese proceso que envolvió la revisión de los postulados profesionales alcanzó distintos segmentos, incluso el de los terapeutas ocupacionales; se colocaba fuertemente en debate las responsabilidades de los técnicos en la formación de valores sociales en cómo ejercer un papel político a partir de su práctica, de forma consciente y decidiendo entre el consenso y el disenso, participando, técnica y políticamente, en la lucha por la hegemonía (Gramsci, 1988; Lopes, 1993/1996). Tal revisión de la acción profesional fomentó cuestionamientos, proposiciones y una perspectiva teórica que fundamentó la terapia ocupacional social (Barros, Ghirardi y Lopes, 2002).

La segunda perspectiva nace de otro cuestionamiento, aquel que se refería tanto al saber médico-psicológico como a sus formas reduccionistas de comprender y de lidiar con fenómenos que se colocaban bajo el binomio "salud-enfermedad", con base en la disciplinarización e institucionalización de problemas sociales, o sea, para ser tratado desde el punto de vista de determinados valores dominantes, cuyo objetivo es el control y la supresión de la libertad de sujetos, individuales y colectivos (Barros, Ghirardi y Lopes, 1999). Se discutía la cantidad en que esos valores impregnaban las disciplinas médicas/psicológicas/clínicas con fuerte influencia de la psiquiatría, pero también se discutían otros abordajes de la propia psicología, que articulaban una serie de proposiciones que van a moldear la acción de los técnicos.

Así, es teóricamente destacable, para la terapia ocupacional social, la comprensión acerca de la desigualdad que emerge de las contradicciones sociales, en las sociedades capitalistas, resultantes de la relación capital-trabajo y de la exploración y precarización producidas por ella. A mediados de la década de 1990, se profundiza en Brasil la flexibilización y desregulación del trabajo en el mundo de la automatización y, en ese sentido, la creciente presencia de grandes contingentes de población viviendo en formas e intensidades variadas, procesos de disolución de los vínculos sociales, de la vulneración de las redes sociales de soporte (Castel, 1998, 1994) y ocasionando una reconfiguración de la cuestión social, también entre nosotros. 
Se resalta que esa reconfiguración se percibirá a finales de la década de 1990, por parte de los terapeutas ocupacionales, presentándose como demandas contemporáneas y con relación a las cuales es posible contribuir, por tanto, exigiendo la construcción de tecnologías específicas (Lopes, Malfitano, Silva, Borba y Hahn 2012). Los sujetos que debido a las transformaciones sociales están expuestos directamente al trabajo precario, a la vulnerabilidad, son colocados al margen, viviendo procesos de ruptura de las redes sociales de soporte y se convierten en foco de la terapia ocupacional social.

En concordancia con el sociólogo francés Robert Castel $(1994,1998)$, podemos encarar las situaciones de privación como un efecto resultante de la conjunción de dos ejes: el de la relación de trabajo (con una gama de posiciones desde el empleo estable a la ausencia completa de trabajo, pasando por formas precarias e intermitentes de ocupación) y el de la inserción relacional (también con un abanico de posiciones entre la inscripción en las redes sólidas de sociabilidad y el aislamiento social total). El desglose de esos dos ejes circunscribe zonas diferentes del espacio social: zona de integración -donde se dispone de garantías de un trabajo permanente y se pueden movilizar soportes relacionales sólidos; zona de desafiliación- que conjuga ausencia de trabajo y aislamiento social, implicando una doble ruptura de las redes de sociabilidad y participación; zona de vulnerabilidad, que asocia trabajo precario y fragilidad relacional, es decir, de la vulnerabilidad, que se expande en función de la coyuntura económica, de las situaciones de guerra (declaradas o "silenciosas" como conocemos en Brasil), de la miseria y de la escasez (Castel, 1994, 1998; Lopes, 2007).

La zona de vulnerabilidad social se presenta en la perspectiva del trabajo y de las redes, sobre aquellos que, además de no tener trabajo, tampoco disponen de las redes sociales de soporte, comprendidas como aquellas provenientes de las relaciones sociales más próximas, desde la familia y los vecinos, hasta aquellas resultantes de la configuración y del acceso a bienes y servicios de protección social (estos últimos integrando lo que Castel (1998) denomina como zona de asistencia). Si no dispone de "empleo/trabajo", pero tiene acceso a redes sociales en torno del eje de inserción -la familia, la comunidad y los servicios de protección - eso puede evitar su desafiliación. Desde el punto de vista de la inserción social, si "apenas" tiene la renta proveniente del trabajo, la vulnerabilidad es de otro orden, pues, tener disponibilidad económica es fun- damental. Sin embargo, eso también se presenta como una dificultad que puede representar la inexistencia de una sociabilidad primaria, algo a lo que no se puede acceder por medio del consumo, ya que ese otro "mercado" gira en torno de carencias afectivas, personales y culturales de los sujetos.

Por lo anterior, para la terapia ocupacional social resulta pertinente comprender que:

- La disciplina refleja lo que sus profesionales piensan y producen, y la forma como se posicionan políticamente frente a las cuestiones sociales.

- $\quad$ Sus métodos están condicionados a determinadas problemáticas que se perciben e incorporan como pertinentes y, para esas áreas de problemas, se articulan posibles soluciones.

- La desigualdad y la pobreza son problemáticas relevantes en el seno de la cuestión social brasileña, adquiriendo configuraciones que requieren una revisión de la formación, de las profesiones y de las pertenencias del papel profesional.

- La terapia ocupacional debe contribuir para la evaluación de las cuestiones impuestas por las contradicciones sociales y culturales.

- El terapeuta ocupacional necesita una formación que lo habilite para trabajar problemáticas que emergen de conflictos sociales, y también, que emergen de los problemas de una sociedad marcada por las desigualdades.

Se deduce, entonces, la necesidad de echar mano de conceptos relativos al entendimiento de las dinámicas de las negociaciones sociales, de incorporar conocimientos socio-antropológicos a los conocimientos específicos, y de invertir tanto en acciones de carácter individual y colectivo como en acciones transdisciplinares, interprofesionales, intersectoriales, etc. En fin, la construcción de aquello que va Ilamando a la acción en el campo social y a la terapia ocupacional social (Barros, Lopes y Galheigo, 2007).

Se configura así, la oportunidad de un diálogo en torno de la creación, proposición y análisis acerca de lo que se ha de hacer en ese campo.

El proyecto METUIA - Grupo interinstitucional de estudios, formación y acciones por la ciudadanía de 
niños, adolescentes, jóvenes y adultos en procesos de ruptura de las redes sociales de soporte-fue importante como articulador de acciones y reflexiones acerca del campo. Creado en 1998, por docentes de terapia ocupacional de la PUC-Campinas, de la UFSCar y de la USP, su propuesta ha sido la de desarrollar proyectos en el ámbito de la enseñanza, investigación y extensión en terapia ocupacional social. Esta disciplina, defendida por METUIA, propone y desarrolla acciones territoriales comunitarias, dirigidas a las poblaciones vulnerables socialmente y en procesos de desafiliación (Barros, Lopes, Galheigo y Galvani, 2007).

Sin embargo, en ese final del siglo XX e inicio del $\mathrm{XXI}$, las problemáticas sociales, resultantes del cambio de los sistemas protectores en el mundo más desarrollados, económica y socialmente desde el punto de vista de la construcción de la esfera pública y de las protecciones sociales, han Ilevado a muchos otros terapeutas ocupacionales a preocuparse por ellas. Podemos citar como ejemplo el proyecto Terapia Ocupacional Sin Fronteras que, en su calidad de organización no gubernamental, reunió a terapeutas ocupacionales con diversas experiencias y produjo una importante visibilidad con la edición del libro "Occupational Therapy Without Borders: learning from the spirit of survivors" (Kronenberg, Pollard y Simó Algado, 2005), posteriormente editado en español (Kronenberg, Pollard y Simó Algado, 2007).

Se percibe el fortalecimiento de una tendencia a pensar que la intervención del terapeuta ocupacional no puede disociarse de la acción política, técnica, social y cultural; se evidencia que conforme la cuestión social se reconfigura y los problemas sociales dejan de quedarse restringidos a los países periféricos, surge una tendencia que nace de los terapeutas ocupacionales de los países centrales, los cuales comienzan a preocuparse con ella dentro de sus propios territorios.

En el mencionado libro, donde se reúnen varios textos de autores de diferentes países, destacamos dos temáticas, con las cuales se dialoga un poco más, en términos de propuesta para pensar la terapia ocupacional en el campo social o la terapia ocupacional social, que son el "apartheid ocupacional" (Kronenberg y Pollard, 2007) y la "justicia ocupacional" (Townsend y Whiteford, 2007).

Frank Kronenberg y Nick Pollard indican la necesidad de que se aborde la naturaleza política de la terapia ocupacional, a partir de la comprensión de ese profesional como agente social; nos dirige a vivenciar una época de "apartheid ocupacional" con la: segregación de grupos de personas mediante la restricción o negación de su acceso a una participación digna y significativa en las ocupaciones de la vida diaria, basada en la raza, color, discapacidad, procedencia nacional, edad, sexo, orientación sexual, religión, creencias políticas, estatus en la sociedad u otras características. Ocasionado por fuerzas políticas, sus consecuencias sociales, culturales y económicas, sistemáticas y dominantes, ponen en peligro la salud y el bienestar de individuos, comunidades y sociedades (Kronenberg y Pollard, 2007, p. 66).

En diálogo con la noción de "apartheid ocupacional", una vertiente canadiense de la terapia ocupacional acuñó el concepto de "justicia ocupacional". La participación en ocupaciones, desarrolladas en la vida diaria, por medio de abordajes centrados en el "clien-

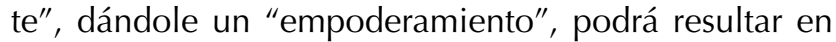
una justicia ocupacional, o sea, en inclusión social (Towsend y Whiteford, 2007).

Salvador Simó Algado, profesor en la Universidad de Vic (Barcelona, España) defiende la posibilidad de una "terapia ocupacional eco-social" que, por medio de la ocupación, lidie con la noción de la sustentabilidad individual, colectiva, indicando la necesidad de articulación de actores sociales, que va desde el alcalde y concejales hasta el empresario de la ciudad, para la realización de acciones terapéutico-ocupacionales centradas localmente (Simó Algado, 2012).

Otro desglose que apareció con bastante fuerza en la $15^{a}$ edición del Congreso Mundial de Terapia Ocupacional de la Federación Mundial de Terapeutas Ocupacional (WFOT, por sus siglas en inglés), que se dio en Chile, en el año 2010, trajo el profesor Alejandro Guajardo de la Universidad Andrés Bello (Santiago de Chile), quien señaló la defensa de una terapia ocupacional crítica que tenía como base los derechos humanos con:

\section{[...] la producción de un nuevo fundamento que la sustente, uno TO de orden social, sea cual sea su ámbitos de acción (salud, educación, justicia, políticas pública, académicas, etc) (Guajardo y Simó Algado, 2010, p.6).}


Por último, con relación a otros interlocutores de la proposición de una terapia ocupacional social, en Brasil tenemos las reflexiones de Maria Isabel Ghirardi, profesora en la Universidad de São Paulo (USP), que constituye la TOPES -Terapia Ocupacional en los Procesos Económicos-Sociales-, trayendo para los terapeutas ocupacionales una visión todavía más focalizada en el ámbito de la economía, del trabajo y en cómo la terapia ocupacional social puede actuar en los procesos económico-sociales más fuertemente (Ghirardi, 2012).

Nos parece esencial ese desglose de proposiciones y proyectos para poder delimitar las perspectivas más actuales en relación al campo social y de la terapia ocupacional.

\section{Proposiciones del Proyecto METUIA}

A lo largo de más de quince años de existencia, varios proyectos han sido desarrollados por docentes, profesionales y estudiantes de terapia ocupacional, en los diferentes núcleos del METUIA. La intervención aplicada que resulta de proyectos de extensión universitaria y de las colaboraciones establecidas en cada uno de ellos, viene sucediendo en espacios públicos, espacios comunitarios e instituciones sociales, como escuelas, albergues, centros comunitarios y otras organizaciones sociales que atienden a poblaciones en proceso de ruptura de redes sociales de soporte.

Concretamente, las experiencias acumuladas por METUIA/UFSCar han producido tecnologías sociales que han sido capaces de fomentar nuevas posibilidades de actuación, integrando y articulando acciones de alcance macro y microsocial (Lopes, Malfitano, Silva, Borba, Takeiti, Garcia y Furlan, 2006; Lopes et al., 2012). En esa perspectiva, destacamos: los Talleres de Actividades, Dinámicas y Proyectos; los Acompañamientos Singulares y Territoriales; la Articulación de Recursos en el Campo Social; y la Dinamización de la Red de Atención (Lopes, Malfitano, Silva y Borba, 2014).

A) Talleres de Actividades, Dinámicas y Proyectos. La terapia ocupacional social utiliza las actividades ${ }^{6}$

6 Se destaca que la nomenclatura "actividades" es ampliamente utilizada en la terapia ocupacional brasileña. Según un estudio de Galheigo y Magalhães (2010), esta terminología fue adoptada desde la llegada de la profesión a Brasil, en la década de 1950, siendo que en los años de 1980 fue resignificada, acoplándosele el sentido de emancipación. A pesar de haber existido y seguir existiendo una ampliación de expresiones para referirse a las actividades, tales como: praxis, hacer, acción humana, ocupación, como un recurso mediador del trabajo de aproximación, acompañamiento, percepción de las demandas y fortalecimiento de los sujetos, individuales y colectivos, para los cuales direcciona su acción. En nuestra experiencia, focalizamos el uso de las actividades en espacios grupales y/o colectivos. Por medio de ese instrumento de trabajo, sobre el cual el terapeuta ocupacional debe ejercer su dominio, se puede conocer el universo inmediato de los sujetos, ampliando significativamente la posibilidad de creación de vínculos y, con eso, generando oportunidades para una actuación profesional que contribuya para la construcción conjunta de planos e proyectos (Lopes, Borba, Trajber, Silva y Cuel, 2011). La utilización de la actividad posibilita el aprendizaje y el reconocimiento de necesidades del sujeto y el desarrollo de la capacidad de éste para buscar soluciones propias y creativas a sus cuestiones.

Se crean, potencialmente, espacios
de experimentación y aprendizaje,
concibiéndose a cada participante
como un ser activo en el proceso de
construcción de la subjetividad, un ser de
praxis, acción y reflexión.

Los talleres (sus actividades, sus proyectos y productos, y sus dinámicas) permiten una gama potente de acciones, que pueden ser clasificadas, comprendidas y aplicadas con distintos propósitos, tales como: - lidiar con las técnicas intrínsecas; - usar y producir materiales/recursos; - transitar por diversos sectores (cultura, arte, deporte, ocio, trabajo, etc.); - conformar propuestas previamente elaboradas con temáticas y objetivos preestablecidos (debates sobre lo cotidiano, perspectivas de vida, intercambios e informaciones sobre el mundo del trabajo, procesos educativos acerca de derechos y deberes, sobre la red de protección a la infancia y adolescencia en la ciudad, entre otros); - atender las necesidades y posibilidades de la vida cotidiana; - mostrar los diferentes sentidos y significados que los sujetos en acción pueden designar o imprimir según sus vivencias personales; en ese caso, aunque las propuestas tengan indicaciones o direccionamientos

cotidiano; el "uso de actividades", o sea, la utilización de recursos de mediación para la ejecución del trabajo del terapeuta ocupacional, sigue siendo predominante en Brasil. 
previos, el interés está en la percepción singular que aquella experiencia proporciona al participante de la acción (Silva, 2011).

Esos dispositivos permiten un contacto más próximo con los jóvenes, a partir del cual se torna posible profundizar en la lectura de las necesidades individuales y colectivas; también promueve un mayor contacto y convivencia entre los propios participantes; proporciona la experimentación de un placentero espacio de sociabilidad e intercambio que puede extrapolar el espacio físico del Taller y trascender para el contexto más amplio (Lopes et al., 2011).

B) Los Acompañamientos Singulares y Territoriales se uti-

lizan en la terapia ocupacional social como una estrategia de intervención que posibilita una percepción e interacción más real del cotidiano y del contexto de vida de los individuos, interconectando sus historias y caminos, su situación actual y su red de relaciones. Tales acompañamientos parten de la escucha atenta a las demandas de personas, grupos o colectivos en dirección de su evaluación, la mayoría de las veces determinadas por la situación de vulnerabilidad, desigualdad social y falta de acceso a servicios sociales y bienes esenciales (Lopes, Borba y Cappellaro, 2011; Malfitano, Adorno y Lopes, 2011).

C) La Articulación de Recursos en el Campo Social comprende una gama de acciones realizadas desde el plano individual, pasando desde los grupos, colectivos, hasta los niveles de la política y gestión; la estrategia está en manejar las prácticas en diferentes niveles de atención en torno de los objetivos comunes y en utilizar los recursos posibles, comprendidos como dispositivos financieros, materiales, relacionales, afectivos, sean estos micro o macrosociales, para componer las intervenciones. Por lo tanto, es necesario disponer de metodologías de intervención que también estén involucradas en esos diferentes niveles, para que sean posibles la identificación, la negociación y la efectiva contribución de dichos recursos (Silva, 2011).

D) La Dinamización de la Red de Atención, en el caso de la atención al público juvenil, se dirige a mapear, divulgar y consolidar todos los programas, proyectos y acciones orientadas para ese grupo poblacional y/o su comunidad, con el intento de fomentar la interacción e integración entre ellos, articulando los diferentes sectores y niveles de intervención, facilitando la efectividad y el direccionamiento de las estrategias (Silva, 2011).
Esas son las marcas que venimos intentando implementar, discutir, llevar para el campo, mejorar la conversación con los terapeutas ocupacionales en portugués, inglés y español; se trata de un conjunto de procedimientos y de recursos que han producido aportes para la acción de una terapia ocupacional social fundada en la dimensión territorial y comunitaria, con vista a una contribución técnica radicada localmente al enfrentamiento de los desafíos puestos para aquellos que se concentran sobre el campo social.

En nuestra experiencia, esa dimensión se construye en la medida en que se reflexiona y se teoriza acerca de las prácticas concretizadas en el cotidiano, a partir del mejor entendimiento de las múltiples dimensiones de las temáticas aportadas por los "quehaceres" de los proyectos.

Antes de todo, es necesario que se piense con respecto a la intervención, es necesario pensar la experiencia junto con la reflexión, pensar y hacer, pensar la experiencia, hacer la reflexión, hacer la experiencia y reflexionar. O sea, el legado de Paulo Freire (1979a; 1979b) continua existiendo en nosotros, para que salgamos del activismo y, al mismo tiempo, no nos atrapemos en la retórica, en la búsqueda de la integración posible entre acción y teoría, experiencia y reflexión.

\section{REFERENCIAS BIBLIOGRÁFICAS}

barros, D. D., Lopes, R. E. \& Galheigo, S. M. (2007). Terapia ocupacional social: concepções e perspectivas. In: A. Cavalcanti y C. Galvão (Eds.). Terapia ocupacional: fundamentação e prática, (pp. 347353). Rio de Janeiro, RJ, Brasil: Guanabara Koogan.

Barros, D. D., Ghirardi, M. I. G. \& Lopes, R. E. (2002). Terapia ocupacional social. Revista Terapia Ocupacional Universidade São Paulo, 13 (3), 95-103.

Barros, D. D., Ghirardi, M. I. G. \& Lopes, R. E. (1999). Terapia ocupacional e sociedade. Revista Terapia Ocupacional Universidade São Paulo, 10 (2/3): 71-76.

Barros, D. D., Ghirardi, M. I. G. \& Lopes, R. E. (2007). Terapia ocupacional social: una perspectiva sociohistórica. In: F. Kronenberg, S. Simó Algado \& N. Pollard. (Eds.) Terapia ocupacional sin fronteras: aprendiendo del espíritu de supervivientes, (pp.141-153). Buenos Aires, Argentina: Madrid, España: Médica Panamericana.

Barros, D. D., Lopes, R. E., Galheigo, S. M. \& Galvani, D. (2007). El Proyecto Metuia en Brasil: ideas y acciones que nos unen. In: F. Kronenberg, S. Simó Algado \& N. Pollard. (Eds.) Terapia ocupacional sin fronteras: aprendiendo del espíritu de supervivientes, 
(pp.392-403). Buenos Aires, Argentina: Madri, España: Médica Panamericana.

Bregalda, M. M. \& Lopes, R. E. (2013). A atuação dos terapeutas ocupacionais no Instituto Nacional do Seguro Social. In: A. P. Simonelli \& D. S. Rodrigues (Eds.). Saúde e trabalho em debate: velhas questões, novas perspectivas, (pp. 293-322). Brasília, DF, Brasil: Paralelo 15.

Castel, R. (1994). Da indigência à exclusão, a desfiliação: precariedade do trabalho e vulnerabilidade relacional. In: A. Lancetti (Ed.). SaúdeLoucura 4 (pp. 21-48). São Paulo, SP, Brasil: Hucitec.

Castel, R. (1998). As metamorfoses da questão social: uma crônica do salário. Petrópolis, RJ, Brasil: Editora Vozes.

Escorel, S. (1999). Vidas ao léu: trajetórias de exclusão social. Rio de Janeiro, RJ, Brasil: Fiocruz.

Francisco, B. R. (1988). Terapia ocupacional. Campinas, SP, Brasil: Papirus.

Freire, P. (1979a). Pedagogia do oprimido. 7a. ed. Rio de Janeiro, RJ, Brasil: Paz e Terra.

Freire, P. (1979b). Educação como prática da liberdade. 9a. ed. Rio de Janeiro RJ, Brasil: Paz e Terra.

Galheigo, S. M. (1997). Da adaptação psicossocial à construção do coletivo: a cidadania enquanto eixo. Revista de Ciências Médicas PUCCAMP, 6 (2/3), 105-108.

Ghirardi, M. I. G. (2012). Terapia Ocupacional em processos econômiCo-sociais. Cadernos de Terapia Ocupacional da UFSCar, 20, 1720. DOI: $10.4322 /$ cto. 2012.0

Gramsci, A. (1988). Os intelectuais e a organização da cultura. 6a. ed. Rio de Janeiro, RJ, Brasil: Civilização Brasileira.

Guajardo, A. \& Simó Algado, S. (2010). Una terapia ocupacional basada en los derechos humanos. TOG (A Coruña), 7 (12), 1-25. Obtenido en 6 de Febrero de 2012, desde: http://www.revistatog.com/ num12/pdfs/maestros.pdf.

Kronenberg, F. \& Pollard, N. (2007). Superar el apartheid ocupacional: exploración preliminar de la naturaleza política de la terapia ocupacional. In: F. Kronenberg, S. Simó Algado \& N. Pollard. (Eds.) Terapia ocupacional sin fronteras: aprendiendo del espíritu de supervivientes, (pp.58-84). Buenos Aires, Argentina: Madrid, España: Médica Panamericana.

Kronenberg, F., Simó Algado, S. \& Pollard, N. (Eds.) (2005). Occupational therapy without borders: Learning from the spirit of survivors. Toronto, ON, Canada: Elsevier Churchill Livingstone.

Kronenberg, F., Simó Algado, S. \& Pollard, N. (Eds.) (2007). Terapia ocupacional sin fronteras: aprendiendo del espíritu de supervivientes. Buenos Aires, Argentina: Madri, España: Médica Panamericana.

Lopes R.E., Malfitano A.P.S., Silva C.R., Borba P.L.O. \& Hahn M.S. (2012). Occupational Therapy Professional Education and Research in the Social Field. WFOT Bulletin, 66, 52-57.
Lopes, R. E. (1990). Currículo mínimo para terapia ocupacional: uma questão técnico-ideológica. Revista de Terapia Ocupacional da Universidade de São Paulo, São Paulo, 1 (1), 33-41.

Lopes, R. E. (1993/1996). A direção que construímos: algumas reflexões sobre a formação do terapeuta ocupacional. Revista Terapia Ocupacional Universidade São Paulo, 4/7, 27-35.

Lopes, R. E. (2007). Rede social de suporte. In: M. B. Park; R. S. Fernandes \& A. Carnicel (Eds). Palavras-chave em educação não-formal, (pp. 249-250). Holambra y Campinas, SP, Brasil: Editora Setembro y Universidade Estadual de Campinas.

Lopes, R. E., Borba, P. L. O., Trajber, N. K. A., Silva, C. R. \& Cuel, B. T. (2011). Oficinas de Atividades com Jovens da Escola Pública: tecnologias sociais entre educação e terapia ocupacional. Interface, 15 (37), 277-288. DOI: 10.1590/S1414-32832011000100021.

Lopes, R. E., Malfitano, A. P. S., Silva, C. R. \& Borba, P. L. O. (2014). Recursos e tecnologias em terapia ocupacional social: ações com jovens pobres na cidade. Cadernos de Terapia Ocupacional da UFSCar, 22 (3), 591-602.

Lopes, R. E., Malfitano, A. P. S., Silva, C. R., Borba, P. L. O., Takeiti, B. A., Garcia, D. B. \& Furlan, P. G. (2006). Terapia ocupacional social e a infância e a juventude pobres: experiências do Núcleo UFSCar do Projeto METUIA. Cadernos de Terapia Ocupacional da UFSCar, 14 (1), 5-14.

Lopes, R. E.; Borba, P. L. O. \& Cappellaro, M. (2011). Acompanhamento individual e articulação de recursos em terapia ocupacional social: compartilhando uma experiência. O Mundo da Saúde, 35 (2), 233-238.

Magalhães, L. \& Galheigo, S. M. (2010). Enabling Internacional Communication among Brazilian Occupational Therapists. Occupactional Therapy Internacional, 17, 113-124.

Malfitano, A. P. S.; Adorno, R. C. F. \& Lopes, R. E. (2011). Um relato de vida, um caminho institucional: juventude, medicalização e sofrimento sociais. Interface, 15 (38), 701-714. DOI: http: 10.1590/ S1414-32832011005000042.

Pinto, J. M. (1979). Relato de uma experiência de terapia ocupacional no campo social. Trabajo presentado en lo V Encontro Científico Paulista de Terapeutas Ocupacionais, São Paulo, SP, Brasil.

Pinto, J. M. (1987). De terapeuta ocupacional para terapeuta ocupacional: os métodos de terapia ocupacional e suas elaborações na UFSCar (1983-1987). Manuscrito no publicado, São Carlos, SP, Brasil.

Pinto, J. M. (1990). As correntes metodológicas em terapia ocupacional no Estado de São Paulo (1970-1985). Tesis de Maestría no publicada, Universidade Federal de São Carlos, São Carlos, SP, Brasil.

Rafante, H. C. \& Lopes, R. E. (2009). Helena Antipoff e a educação dos excepcionais: uma análise do trabalho como princípio educativo. Revista HISTEDBR On-line, 33, 1-24. Obtenido en 6 de Junio de 2014, desde: http://www.histedbr.fe.unicamp.br/revista/edicoes/33/art15_33.pdf. 
Silva, C. R. (2011). Percursos juvenis e trajetórias escolares: vidas que se tecem nas periferias das cidades. Tesis Doctoral. Universidade Federal de São Carlos, São Carlos, SP, Brasil.

Simó Algado, S. (2012). Terapia Ocupacional eco-social: hacia una ecología ocupacional. Cadernos de Terapia Ocupacional da UFSCar, 20, 7-16. DOI: 10.4322/cto.2012.001.

Soares, L. B. T. (1991) Terapia ocupacional: lógica do capital ou do trabaIho? São Paulo, SP, Brasil: HUCITEC.

Townsend, E. \& Whiteford, G. (2007). Uma estrutura de participación em el marco da la justicia ocupacional: processos prácticos basados em la comunidad. In: F. Kronenberg, S.

Simó Algado \& N. Pollard. (Eds.) Terapia ocupacional sin fronteras: aprendiendo del espíritu de supervivientes, (pp.110-126). Buenos Aires, Argentina: Madrid, España: Médica Panamericana.

Ventura, Z. (1994). Cidade Partida. São Paulo, SP, Brasil: Companhia das Letras. 